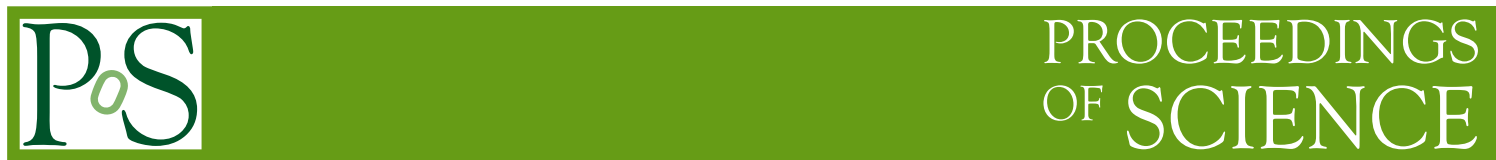

\title{
Prospects for the study of heavy-ion collisions at the NICA collider at JINR
}

\section{I. Kolesnikov for the NICA Collaboration*}

Joint Institute for Nuclear Research, Dubna, 141980 Russia

E-mail: Vadim.Kolesnikov@cern.ch

The Nuclotron-based Ion Collider fAcility (NICA) is now under construction at JINR (Dubna, Russia). A rich nomenclature of high intensity heavy ion beams in the energy range $4<\sqrt{s_{N N}}<$ $11 \mathrm{GeV}$ will become available by about 2020. The main physics goal of the NICA project is the experimental exploration of the properties of high density nuclear matter with an emphasis to the nature of the deconfinement phase transition, in-medium particle properties, and search for the QCD critical point. Two experiments: MPD and BM@N are foreseen for study heavy-ion collisions in the collider mode and fixed target configuration, respectively. This report summarizes the current status of the accelerator and detector projects.

EPS-HEP 2017, European Physical Society conference on High Energy Physics

5-12 July 2017

Venice, Italy

${ }^{*}$ Speaker. 


\section{Introduction}

Heavy-ion collisions is the only experimental method of creating in the laboratory nuclear matter under extreme conditions of density and temperature. Such experiments provide unique perspectives of resolving the most fundamental problems of the underlying theory - confinement and chiral symmetry breaking. Experimental data about the nature of existing in the QCD matter phase transformations are scarce, especially in the region of the highest baryonic density. For the purpose of filling this gap, a new accelerator facility NICA [1] is deigned, which provide a broad range of research opportunities. The NICA scientific goals include the study of the equation-ofstate (EoS) at high baryon density, properties of the hadron spectral function, features of hyperonnucleon interaction in the medium, critical behavior of the QCD matter, and the spin structure of the nucleon. In order to archive these goals a detailed energy and system size scan will be performed in the energy range $4<\sqrt{s_{N N}}<11 \mathrm{GeV}$ with an emphasis to the production of multiple hadron species, light nuclei, as well as electrons and gammas. The first round of NICA experiments will concentrate on a variety of diagnostic observables with an experimental setup to be optimized for the study of the excitation function of event-by-event fluctuations and correlations of bulk event properties. To obtain a proper normalization for the measured particle distributions from heavyion collisions, a study of elementary reactions is foreseen as well. The energy range at the NICA collider will be extended to the lower energy region with the Nuclotron accelerator extracting beams focusing on the investigation of the reaction dynamics, in-medium hadron properties, production of multistrange hyperons at the threshold, and search for hypernuclei.
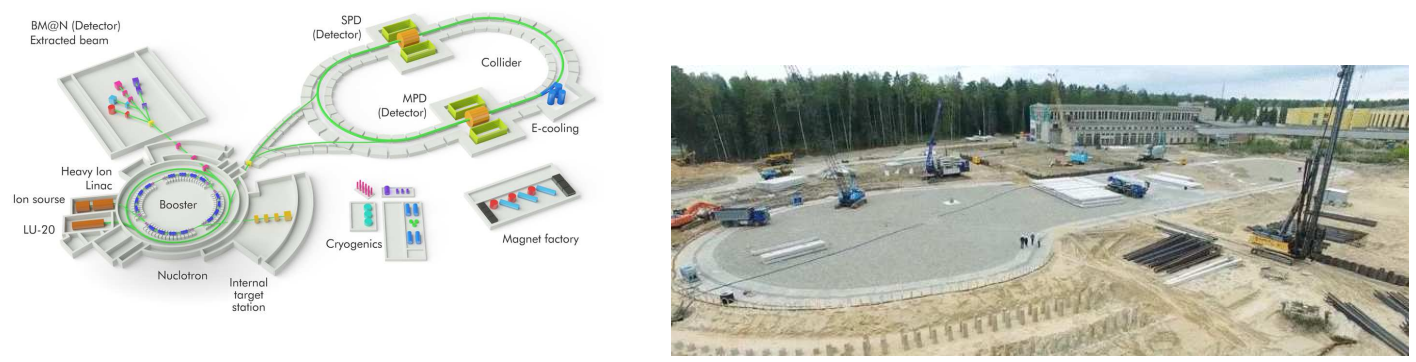

Figure 1: Left: A scheme of the NICA complex. Right: An image of the NICA construction site.

\section{Progress of construction and installation of the NICA complex}

The NICA accelerator facility (see Fig. 1, left) consists of an injector, a booster, the upgraded Nuclotron accelerator, and two collider storage rings [1]. The NICA injector, which is designed and fabricated in cooperation with German and Russian research companies, will provide a variety of ion species up to gold nuclei with a design intensity of $2 \cdot 10^{9}$ particles per cycle. It consists of a heavy-ion source followed by a heavy-ion linac (HILAC) and a RFQ fore-injector. The booster synchrotron with its magnetic ring to be located inside of the existing yoke of the Dubna Synchrophasotron will accelerate ions up to $600 \mathrm{MeV} /$ nucleon energy. Its the most challenging design characteristics are ultrahigh vacuum and electron cooling. The upgraded Nuclotron should 
provide $p, d$, and heavy-ion beams with the maximum energy per nucleon of $5.8 \mathrm{GeV}$ for $\mathrm{A} / \mathrm{Z}=0.5$ specie and $4.5 \mathrm{GeV}$ for ${ }^{+79} \mathrm{Au}$ nuclei. Two NICA collider rings of about $503 \mathrm{~m}$ circumference each are based on double-aperture superconductive magnets being designed in our Laboratory and providing the maximum field up to $1.8 \mathrm{~T}$. Given to the modern electron and stochastic cooling systems at NICA, the achieved collider luminosity for gold ions will be of the order of $10^{27} \mathrm{~cm}^{-2} \mathrm{~s}^{-1}$. In addition, the NICA accelerator will provide beams of polarized protons and deuterons up to center-of-mass energy of $27 \mathrm{GeV}$ with the luminosity of $10^{32} \mathrm{~cm}^{-2} \mathrm{~s}^{-1}$.

Serial fabrication of the magnets for the NICA facility is scheduled for 2016-2018 and for the time being one-third of all the dipoles for the booster has being constructed and tested. The construction of the new NICA infrastructure, including buildings, transfer lines, service and supply systems, is progressing according to plan. For example, more than $80 \%$ of the concrete work for the NICA and detector buildings has done (see Fig. 1, right). The process of commissioning of the collider elements is scheduled for 2018-2019 and the first heavy-ion collision at the NICA collider is expected in 2020 with a start-up version of the project (i.e. the peak luminosity reduced to the level of $5 \cdot 10^{25} \mathrm{~cm}^{-2} \mathrm{c}^{-1}$ ). The completion of the NICA commissioning with its nominal luminosity value is planned for 2023 .

\section{Status of the experiments at NICA}
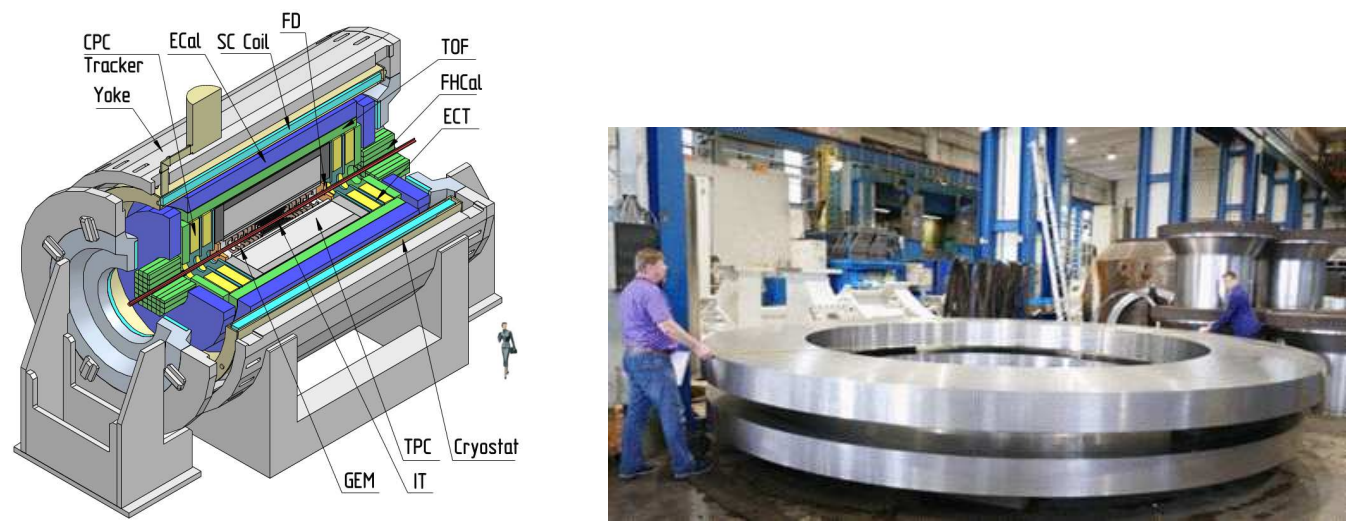

Figure 2: Left: A view of the MPD detector at the NICA collider. Right: A support ring of the MPD magnet yoke.

Study of heavy-ion collisions at the NICA collider will be performed with the MultiPurpose Detector (MPD) which is designed as a large uniform acceptance spectrometer capable of detecting hadrons, electrons and gammas. MPD consists of layers of subdetectors (see Fig.2, left panel) inside a superconducting solenoid generating a magnetic field of up to $0.6 \mathrm{~T}$. The magnetic field inhomogeneity inside the tracking volume will be less than $10^{-3}$ in order to ensure the good tracking resolution for the transverse momentum range up to several $\mathrm{GeV} / \mathrm{c}$. The main tracking device of the MPD detector - cylindrical Time-Projection Chamber (TPC) of $2.8 \mathrm{~m}$ (diameter) by $3.4 \mathrm{~m}$ (length) will provide high efficiency track reconstruction within the pseudorapidity range $|\eta|<2$. In addition, with 53 measured space points per a track at midrapidity, TPC will enable particle identification via the specific energy loss $(d E / d x)$ measurement with a precision better than $8 \%$. 
At larger pseudorapidities TPC tracking will be supplemented by a multi-layer straw tube tracker (ECT). The Inner Tracker (IT), which will be made of 4-6 layers of double-sided silicon microstrip detectors, could provide precision tracking points allowing determination of the position of secondary decay vertices displaced from the primary vertex. A Multigap Resistive Plate Chambers system of an intrinsic time resolution better than $80 \mathrm{ps}$ is devoted to charge particle identification in the range of momentum up to $3 \mathrm{GeV} / \mathrm{c}$. An Electromagnetic Calorimeter (ECAL) for electron and gamma measurements located behind the TOF detector will be built of more than 40000 of lead-scintillator sampling towers of 18 radiation length thickness and of about $3 \mathrm{~cm}^{2}$ cross section. As the results of beam test indicate, an energy resolution of $5 \%$ can be achieved for electrons with the energy of $1 \mathrm{GeV}$ for such a module. Arrays of quartz counters (FD) will provide fast timing and triggering signal. Two arms of hadron calorimeters (FHCAL) covering the peudorapidity range $2.5<|\eta|<4$ will measure the deposit energy of spectators providing data for the offline centrality estimate and event plane analysis. A more detailed description of the detector components can be found elsewhere [2].

After completing of R\&D and preparation of Technical Design reports the MPD Collaboration is now in the process of industrial production of detector elements. For example, the unique MPD superconductive magnet is under production in Genoa, Italy and Katowice, Czech Republic (see Fig. 2, right panel) such that a first control assembling of the magnet yoke is scheduled for October 2017.
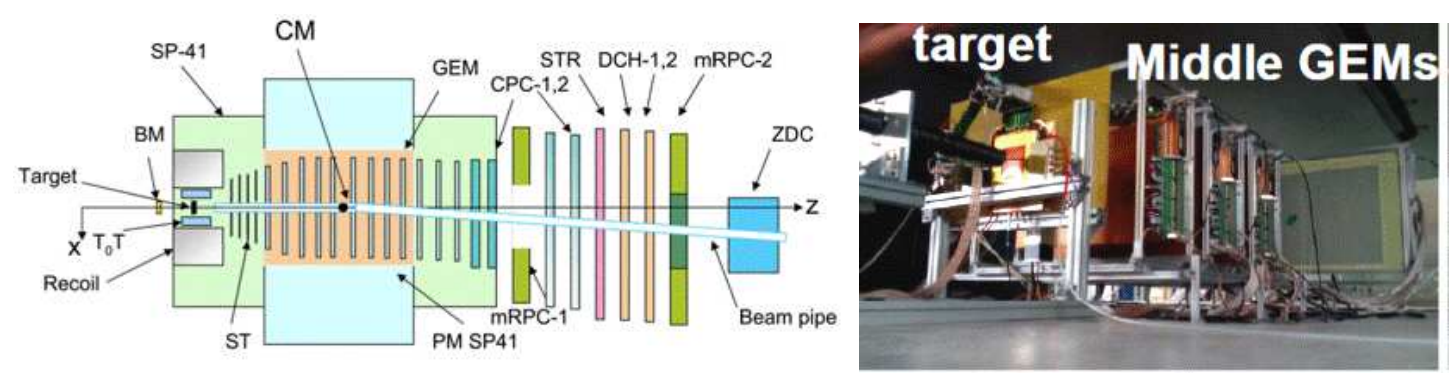

Figure 3: Left: A scheme of the BM@N experiment at the Nuclotron. Right: A fraction of the BM@N tracking system intalled on the beam line.

BM@N (Baryonic Matter at Nuclotron) is a fixed target experiment at the Nuclotron accelerator [3], a sketch of its experimental set-up is shown in Fig. 3 (left panel). The charged track trajectories will be measured with a set of 12 Gaseous Electron Multipliers (GEM) chambers located downstream of the target inside an analyzing magnet of $0.8 \mathrm{~T}$ and drift/pad detectors ( $\mathrm{DCH}, \mathrm{CPC}$ ) situated outside the magnet. Time-of-flight detectors (TOF1,2) made of Resistive Plate Chambers with a strip read-out are designed for discrimination between particle species up to a few $\mathrm{GeV} / \mathrm{c}$ momentum. The recoil detector at the backward hemisphere is designed for event plane determination. Zero degree calorimeter (ZDC) is constructed to measure the energy of forward going particles and calculate the degree of collision centrality.

All the GEM detectors for the BM@N experiment have been constructed in a close collaboration of physicists from JINR and CERN. Several first produced GEMs were recently tested with beams of carbon and deuterons at the Nuclotron. The existing configuration of the central tracker includes six half-sized GEM detectors installed along the beam line (see Fig. 3 right panel); it will 
be extended further up to the full configuration in 2019. At present, the team is in preparation for beginning of data taking in 2017 with a krypton beam. The experimental program will be continued in 2019 with gold nuclei.

\section{Summary}

In this report, an overview of the current status of the NICA project realization is given. The realization plan foresees a staged construction and commissioning with the goal to start the BM\&N experiment with the Nuclotron extracted beams in 2017 and continuation of the research program at higher energies after putting the startup configuration of the NICA collider into operation in 2020.

\section{References}

[1] V. D. Kekelidze, R. Lednicky, V. A. Matveev, I. N. Meshkov, A. S. Sorin and G. V. Trubnikov, Eur. Phys. J. A (2016) 52: 211.

[2] V. Golovatyuk, V. Kekelidze, V. Kolesnikov, O. Rogachevsky, and A. Sorin, Eur. Phys. J. A (2016) 52: 212.

[3] Mikhail Kapishin for the BM@N Collaboration, Eur. Phys. J. A (2016) 52: 213. 